\section{Ferromagnetismus in der Eisengruppe} Von Franz Bader

Institut für theoret. und angew. Physik der Technischen Hochschule und Max-Planck-Institut für Metallforschung, Abt. Prof. U. Dehlinger, Stuttgart

(Z. Naturforschg. 8 a, 334 [1953]; eingeg. am 23. März 1953)

Entsprechend der von Ganzhorn ${ }^{1}$ gruppentheoretisch entwickelten homöopolaren Auffassung verteilen sich in einem kubischen Kristall die ersten fünf 3 d-Elektronen auf ein unteres Dreier- und Zweierband $\left(\mathrm{d}^{3}\right.$ bzw. $\left.\mathrm{d}^{2}\right)$ mit je 3 bzw. 2 Elektronen/Atom, die mit Nachbarn 1. bzw. 2. Sphäre bindende Überlappungen mit antiparallelem Spin eingehen. Bei $\mathrm{Cr}$ sind diese Bänder gerade aufgefüllt. Die restlichen 5 d-Elektronen besetzen von $\mathrm{Mn}$ ab das obere $\mathrm{d}^{3}$ - und $\mathrm{d}^{2}$-Band, wo sie mit entsprechenden Nachbarn lokkernde Bindungen mit parallelem Spin eingehen und somit Magnetismus hervorrufen können. Infolge der Austauschenergie (1. Näherung) stellen sich diese Elektronen in den schmalen d-Bändern parallel, soweit es mit dem Pauli-Prinzip, welches auch im Kristall für das Einzelatom Gültigkeit haben soll, vereinbar ist. Wegen der Doppelbesetzung jeden Zustandes nehmen das obere Dreier- und Zweier-Band maximal 1,5 bzw. 1 Plus-Spin/Atom auf. Darüber hinaus müssen mit zunehmender Elektronenzahl Minus-Spins eintreten, welche das magnetische Moment wieder gleichmäßig erniedrigen. Daraus folgt zwangsläufig das Maximum der Sättigungsmagnetisierung in der raumzentrierten Fe-Co-Legierung mit 2,5 $\mu_{\mathrm{B}}$ /Atom (gemessen 2,46 $\mu_{\mathrm{B}}{ }^{2}$; Abb. 1). Das vorzeitige Umbiegen der Kurve bei der Fe-Ni-Legierung erklärt sich aus dem homöopolaren Charakter des benutzten Modells.



Abb. 1. Sättigungsmagnetisierung in Bohrschen Magnetonen pro Atom über der Elektronenzahl aufgetragen. Gestrichelte Gerade, sowie Gerade (a) und (b): s. Text.

${ }^{1}$ K. Ganzhorn, Stuttgarter Diplomarbeit 1950; Stuttgarter Dissertation 1952; Z. Naturforschg. 7a, 291-292 [1952].

${ }^{2}$ E. C. Stoner, Rep. Progr. Physics 11, 43-112 [1948].
Da im flächenzentrierten Gitter von 12 nächsten Nachbarn höchstens 8 entgegengesetzten und mindestens 4 parallelen Spin aufweisen, sättigen sich aus den Dreierbändern 1 bindendes und 1 lockerndes Elektron im Atom selbst ab, so daß die oberen Bänder zusammen nur mit maximal 2 Plus- und 2 MinusSpins besetzt werden können. Die Auffüllung dieser oberen Bänder erfolgt deshalb im flächenzentrierten Gitter längs der gestrichelten Geraden der Abb. 1 bis zum Maximum $2 \mu_{\mathrm{B}}$ (gemessen etwa 1,94 $\mu_{\mathrm{B}}$ ). Auf dieser Kurve liegt $\gamma$-Fe. Wo die raumzentrierte Kurve diesen flächenzentrierten Zweig trifft, hat die magnetische Energie, welche die raumzentr. Phase stabilisiert, soweit abgenommen, daß bei $T=0$ die flächenzentr. Phase stabil wird, zumal bei ihr in den oberen lockernden Bändern 1 Elektron weniger auftritt. Die zunächst theoretisch postulierte, gestrichelte Gerade der Abb. 1 ist experimentell bestätigt ${ }^{3}$.

Links von den beiden Umkehrpunkten ist antiferromagnetisches Verhalten mit dem Pauli-Prinzip verträglich, wobei die unteren Bänder antiferromagnetisch ausgerichtete Teilgitter ausbilden (Neutroneninterferenzen zeigen Antiferromagnetismus bei $\mathrm{Cr}$, $\left.\mathrm{Mn}, \mathrm{Fe}^{4}\right)$. Die antiferromagnetische Wirkung von 0,56 Elektronen/Atom, die bei $\alpha-\mathrm{Fe}$ in beiden Teilgittern gegeneinander ausgerichtet sind, wird aber durch die ferromagnetische Wirkung von 1,22 Elektronen des oberen $\mathrm{d}^{3}$-Bandes, die sich über den ganzen Elementarbezirk hinweg parallel ausrichten, überwunden. Für das flächenzentrierte Gitter gibt die Gerade (a) der Abb. 1 die Zahl der antiferromagnetisch wirksamen Elektronen an, die Gerade (b) die Zahl der Elektronen des oberen $d^{3}$-Bandes, welche über beide antiferromagnetische Teilgitter hinweg parallel ausgerichtet sind. Links vom Schnittpunkt A überwiegt antiferromagnetisches Verhalten $(\gamma$-Fe), rechts Ferromagnetismus. In der flächenzentrierten Fe-Ni-Legierung liegt $\mathbf{A}$ bei etwa $20 \%$ Ni. Eine Extrapolation der gemessenen Curie-Temperatur ergibt in dieser Legierung bei etwa gleicher Konzentration $0^{0} \mathrm{~K}^{3}$.

Ferner zeigt sich in Übereinstimmung mit der Erfahrung ${ }^{5}$, daß bei $\alpha$-Fe die spezifische Wärme der Elektronen über dem Curie-Punkt etwa $4 \mathrm{mal}$ größer ist, als man aus Tieftemperaturmessungen zu erwarten hätte. Dies bedingt das Auftreten der $\delta$-Phase ${ }^{6}$. Für Fe ergibt sich in Übereinstimmung mit der Erfahrung ein positives, für Ni ein negatives Vorzeichen der HallKonstanten.

Aus den entwickelten Vorstellungen heraus kann für das Verhältnis der magnetischen Kopplung bei Co, Fe und Ni 2,193:1,73:1 angegeben werden, während die experimentell gemessenen Curie-Temperaturen sich verhalten wie 2,192:1,66:1. Die geringe Abweichung bei Fe dürfte auf die verschiedene Gitterstruktur zurückzuführen sein.

3 M. Peschard, Revue Métallurgie 22, 581 [1925], Abb. 25, Abb. 36, Kap. IV \& 9.

${ }^{4}$ C. G. Shull, Abstract in Washington Conference on Magnetism, Sept. 1952.

5 E. C. Stoner, J. Physique Radium 12, 372 [1951].

${ }^{6}$ U. Dehlinger, Z. Naturforschg. 8a, 87 [1953]. 